\title{
Estrategias didácticas para el fortalecimiento de la competencia comunicativa lectora a través de textos narrativos dramatizados mediante el teatro callejero.
}

\author{
Didactic strategies for strengthening communicative reading competence \\ through narrative texts dramatised by means of street theatre
}

\author{
Gloria Yaneth Rodríguez-Martínez ${ }^{\mathrm{a}}$, Sandra Milena Riveros-Giraldo ${ }^{\mathrm{b}}$ \\ a*Magister en Educación, gloyaroma12345@hotmail.com, 0000-0001-5591-8933, Colegio Santos Apóstoles, Cúcuta, Colombia
bMagister en Educación, milena-river@hotmail.com, 0000-0002-9462-6447, Colegio Santos Apóstoles, Cúcuta, Colombia
}

Forma de citar: Rodríguez-Martínez, G.Y. Riveros-Giraldo, S.M. Estrategias didácticas para el fortalecimiento de la competencia comunicativa lectora a través de textos narrativos dramatizados mediante el teatro callejero. Eco Matemático, 10 (1), 87-103

Recibido:30 septiembre 2018

Aceptado: 15 diciembre 2018

\section{Palabras clave}

Estrategias didácticas, Competencia comunicativa lectora, Teatro callejero.
Resumen:La educación en los actuales momentos toma un lugar apremiante en torno al desarrollo de estrategias que involucren el uso de la competencia comunicativa lectora a través del teatro callejero. Las realidades que se corresponden con el manejo del área de lenguaje demuestran la necesidad de reestructurar tales situaciones, de este modo, como vía que permita abordar tal realidad, se hizo uso del método cualitativo, a fin de comprender las realidades sociales y educativas tal cual, y como se gestan. De este modo se aplicaron como instrumentos la entrevista y la observación, por tal motivo se pudo corroborar que había la creciente necesidad del desarrollo de las habilidades lectoras y comunicativas. Para ello fue necesario el desarrollo de estrategias pedagógicas fundamentadas en el uso del teatro callejero y poder dar remedio a tal situación y poder ofrecer una educación de calidad, adecuada a las realidades obtenidas del contacto con la realidad educativa la institución educativa colegio los Santos Apóstoles de Cúcuta Norte de Santander. Como conclusiones principales se puede denotar que el uso de estrategias didácticas dinamiza el desarrollo de los procesos formativos y hace que los estudiantes comprendan de una mejor manera los contenidos referidos a la comprensión lectora.

*Autor para correspondencia: gloyaroma12345@hotmail.com 


\section{Keywords \\ Didactic strategies, Communicative reading competence, Street theatre.}

\begin{abstract}
Education in current times takes a pressing place around the development of strategies that involve the use of communicative reading skills through street theater. The realities that correspond to the management of the language area demonstrate the need to restructure such situations, thus, as a way to address such reality, the qualitative method was used, in order to understand the social and educational realities as is, and how they are created. In this way, interview and observation were applied as instruments, for this reason it was corroborated that there was a growing need for the development of reading and communication skills. For this, it was necessary to develop pedagogical strategies based on the use of street theater and to be able to remedy this situation and to be able to offer a quality education, appropriate to the realities obtained from contact with educational reality, the educational institution Colegio los Santos Apóstoles de Cúcuta Norte de Santander. As main conclusions it can be denoted that the use of didactic strategies dynamizes the development of the formative processes and makes the students understand in a better way the contents related to reading comprehension.
\end{abstract}

\section{Introducción}

La comprensión lectora es una habilidad esencial en el desarrollo del lenguaje del individuo, permitiendo su interacción con la realidad y constituyéndose en instrumento por el cual el estudiante se integra a su entorno, ya sea familiar, social o cultural. Al respecto.

"La palabra escrita está presente en todas partes y, por lo tanto, la lectura es una competencia fundamental cada vez más necesaria en todos los ámbitos de la vida. Las diferentes destrezas inherentes a la lectura, incluida la lectura digital, son esenciales para el desarrollo personal y social del individuo, para participar de manera informada y activa en la sociedad y para ejercer con plenitud todos los derechos que se tienen como ciudadano". (EACEA. 2011).

Por lo tanto, se considera que esta competencia brinda a los estudiantes destrezas y habilidades en el desarrollo de la competencia comunicativa lectora a través de la dramatización; en nuestro caso con el teatro callejero a la vez les permite comunicarse, compartiendo libremente con otros en contextos sociales o particulares de manera espontánea. Se puede decir que el desarrollo de esta competencia permite hacer un adecuado uso del lenguaje desde todos sus ámbitos: oral, escrito, interpretativo, comunicativo, entre otros. En este sentido, el lenguaje es una de las capacidades esenciales en el desarrollo evolutivo del ser humano, lo cual es considerado por el MEN en los estándares básicos del área de lengua castellana, porque:

"Forma parte de las características que definen al ser humano como especie única. En este orden de ideas, todos los seres humanos están dotados con la capacidad lingüística y es función primordial de la formación en lenguaje aportar a su adecuado desarrollo. Según esto, se plantea que dicha formación se orienta hacia el enriquecimiento de dimensiones". (MEN, 2006)

De este modo, los estudiantes del Colegio Santos Apóstoles presentan dificultades en los procesos de lectura, evidenciando debilidades en la comprensión, interpretación y argumentación de textos apropiados a su edad y escolaridad. Teniendo en cuenta esta situación y con el propósito de cumplir 
los objetivos planteados para el presente estudio, se pretende diseñar estrategias didácticas que a través del teatro callejero promuevan en los niños y niñas interés y pasión por lo que leen, convirtiéndose en un hábito de vida que les permitirán ser competentes, eficaces y eficientes para su futuro personal, cultural y profesional. Así mismo, que contribuyan al fortalecimiento de la comprensión lectora, y estas se conviertan en herramientas básicas para adquirir, mejorar y consolidar la competencia comunicativa lectora en el Área de Lengua Castellana.

Partiendo de que la base para la adquisición de habilidades en la comprensión lectora se cimienta y profundiza en el primer ciclo de la Educación Primaria, y que el grado tercero es fundamental para determinar el desarrollo de dicha competencia en los estudiantes, se implementaran estrategias pedagógicas para el desarrollo de la competencia comunicativa lectora mediadas por la dramatización en el teatro callejero que las fortalezcan, alcanzando los estándares establecidos para dicho ciclo en el área de lengua castellana. Y de esta manera, lograr mejorar el rendimiento académico, disminuir la mortalidad académica y la deserción escolar; por consiguiente, se logrará aumentar el índice sintético de calidad de la institución educativa a objeto de estudio.

Por otra parte, es de entender que de conformidad con el artículo 67 de la Constitución Política, el Ministerio de Educación Nacional, con el fin de velar por la calidad, por el cumplimiento de los fines de la educación y por la mejor formación moral, intelectual y física de los educandos, un Sistema Nacional de Evaluación de la Educación que opere en coordinación con el Servicio Nacional de Pruebas del Instituto Colombiano para el Fomento de la Educación Superior, ICFES, y con las entidades territoriales y sea base para el establecimiento de programas de mejoramiento del servicio público educativo (p.18).
Este Sistema de Evaluación de la Calidad de la Educación tiene la función de evaluar periódicamente todos los actores y componentes del servicio educativo, entre los cuales se incluyen los resultados de aprendizaje. Es por esta razón que las PRUEBAS SABER, es concebida como una evaluación nacional de calidad educativa y se aplican a estudiantes de $\operatorname{los} \operatorname{grados} 3^{\circ}, 5^{\circ}$ y $9^{\circ}$ en algunas áreas del conocimiento, con el propósito de medir sus logros. Los resultados proporcionan la información necesaria y efectiva para orientar la formulación de políticas y programas que permitan apoyar el mejoramiento de la calidad educativa de las instituciones educativas del país.

"El Sistema diseñará y aplicará criterios y procedimientos para evaluar la calidad de la enseñanza que se imparte, el desempeño profesional del docente y de los docentes directivos, los logros de los alumnos, la eficacia de los métodos pedagógicos, de los textos y materiales empleados, la organización administrativa y física de las instituciones educativas y la eficiencia de la prestación del servicio" (p.18).

Para el presente artículo "Estrategias didácticas para el fortalecimiento de la competencia comunicativa lectora a través de textos narrativos dramatizados mediante teatro callejero" el marco teórico se hace indispensable, puesto que permite conocer todo lo relacionado con el objeto de estudio, de esta forma se puede hacer una formación holística desde la perspectiva de los autores en su mayoría reconocidos con el fin de conocer planteamientos claros que contribuyen de manera concisa al desarrollo metodológico de la investigación.

\section{Fundamentos Teóricos}

\section{Estrategia Didáctica}

El concepto de estrategias didácticas se involucra con la selección de actividades y prácticas pedagógicas en diferentes momentos formativos, 
métodos y recursos de la docencia. Hacer una distinción conceptual, entre método, técnica y estrategia, permite asumir coherentemente el Aprendizaje Colaborativo como una propuesta para los espacios mediados, o de orden tutorial.

La estrategia didáctica es la planificación del proceso de enseñanza aprendizaje para la cual el docente elige las técnicas y actividades que puede utilizar a fin de alcanzar los objetivos propuestos y las decisiones que debe tomar de manera consciente y reflexiva. Al entender que la estrategia didáctica es el conjunto de procedimientos, apoyados en técnicas de enseñanza, que tienen por objeto llevar a buen término la acción pedagógica del docente, se necesita orientar el concepto de técnica como procedimientos didácticos y el recurso particular para llevar a efecto los propósitos planeados desde la estrategia. Las estrategias didácticas apuntan a fomentar procesos de autoaprendizaje, aprendizaje interactivo y aprendizaje colaborativo. (Fonseca, 2007).

\section{Competencias}

Teniendo en cuenta que las competencias es el conjunto de capacidades, conocimientos, habilidades que puede tener el estudiante y que le sirven para resolver problemas de su vida ya sea en sus relaciones interpersonales y cotidianas, según el (MEN, 2015).

"Son los conocimientos, habilidades y destrezas que desarrolla una persona para comprender, transformar y participar en el mundo en el que vive. La competencia no es una condición estática, sino que es un elemento dinámico que está en continuo desarrollo. Puede generar, potenciar apoyar y promover el conocimiento".

El maestro debe diseñar e implementar estrategias pedagógicas que le permitan potenciar el trabajo que hace en el aula y que pueda brindar herramientas de calidad para la enseñanza aprendizaje teniendo en cuenta los referentes comunes establecidos por el MEN, al precisar los niveles de calidad a los que tienen derecho todos los estudiantes del país, es decir los estándares básicos de competencias.

Según (Sarramona, 1998), una competencia se vincula con las capacidades más que con simple saberes, porque el énfasis se pone en el "saber hacer", sin que falten por ello los saberes y las predisposiciones o actitudes que los envuelven. Las actividades competenciales son de carácter complejo, porque las situaciones que se pretenden resolver tienen este cariz (p.13).

\section{Competencia comunicativa - lectora.}

La competencia comunicativa - lectora explora la forma como los estudiantes leen e interpretan diferentes tipos de textos. Se espera que puedan comprender tanto la información explícita como la implícita en los textos, establecer relaciones entre sus contenidos y lo que saben acerca de un determinado tema, así como realizar inferencias, sacar conclusiones y asumir posiciones argumentadas frente a los mismos. En términos generales, la prueba de lectura les propone a los estudiantes una reflexión en torno a qué dice el texto (contenidos -conceptuales e ideológicos-); cómo lo dice (organización); para qué lo dice y por qué lo dice (pragmática); cuándo lo dice y quién lo dice. Las preguntas de la prueba se orientan a establecer la capacidad de los estudiantes para realizar lecturas literales, inferenciales o críticas.

Con base en las orientaciones establecidas en los estándares básicos de competencias, las preguntas que evalúan la competencia comunicativa - lectora se organizan alrededor de textos seleccionados de acuerdo con los siguientes criterios: (a) la pertinencia de la temática en función de la edad de los estudiantes y el grado que cursan; (b) el vocabulario; (c) la complejidad sintáctica; (d) los saberes previos según el grado cursado; (e) la complejidad estilística; (f) 
la complejidad de la estructura del texto; y (g) la extensión. Asimismo, se tienen en cuenta diferentes tipos de textos, atendiendo la diversidad de formas de organización y estructuración de información: (1) Textos continuos organizados en oraciones y párrafos, esto es, escritos en prosa y también en verso; (2) Textos discontinuos como listas, formularios, gráficos o diagramas; (3) Textos mixtos como historieta o cómic. Es importante aclarar que en grado $3^{\circ}$ encontramos cuentos, poemas, rondas o cancioncillas, noticias y afiches. (Colombia Aprende, 2012).

\section{Textos narrativos.}

Según Alamo (2016) el concepto de texto puede entenderse como aquel enunciado o conjunto de ellos que viene a conformarse como un todo organizado, coherente, policodificado y pluriisotópico, de acuerdo con las siguientes características: a) está verbalmente fijado; b) tiene una estructura interna; c) guarda relación con otros textos, enunciados ideoculturales y sistemas e instituciones externas, y d) es susceptible de cualquier tipo de análisis.

Un texto narrativo es una forma de expresión que cuenta hechos o historias acontecidas a sujetos, ya sea humanos (reales o personajes literarios), animales o cualquier otro ser antropomorfo, cosas u objetos; en él se presenta una concurrencia de sucesos (reales o fantásticos) y personas en un tiempo y espacio determinados. Dos elementos básicos de las narraciones son la acción (aunque sea mínima) encaminada a una transformación, y el interés que se produce gracias a la presencia de elementos que generan intriga (definida ésta como una serie de preguntas que porta el texto y a las cuales la narración termina dando respuesta) Adam \& Lorda, (1999).

\section{Teatro callejero}

El teatro callejero se presenta como una estrategia para el fomento de la comunicación a través de las realidades que conforman la cotidianidad de la vida del ser humano, por tal motivo, su uso en la educación se estima como un supuesto metodológico y didáctico que hace énfasis en la conformación de una realidad educativa totalmente distinta y que se apunte al uso de elementos didácticos que ayuden a los estudiantes a mejorar la comprensión del área de lenguaje. En este sentido, González (2007) señala que:

El teatro callejero, incluyendo tecnologías que lo hagan atractivo para esta población, contribuiría a que tanto niños como jóvenes comprendan la importancia de la autoformación, del papel que cumplen en su sociedad y de la importancia de alimentar y apreciar su cultura y sus valores.

\section{Método}

Esta investigación se basa en un enfoque cualitativo, el cual es definido por Blasco \& Pérez, (2007), como aquella que estudia la realidad en su contexto natural y cómo sucede, sacando e interpretando fenómenos de acuerdo con las personas implicadas. Utiliza variedad de instrumentos para recoger información como las entrevistas, imágenes, observaciones, historias de vida, en los que se describen las rutinas y las situaciones problemáticas, así como los significados en la vida de los participantes. Al referirse a la metodología cualitativa como un modo de encarar el mundo empírico, señalan además que en su más amplio sentido esta investigación produce datos descriptivos: las palabras de las personas, habladas o escritas y la conducta observable.

\section{Proceso de la investigación}

El presente trabajo es una investigación acción la cual es definida por (Murillo, 2010) como una forma de indagación auto reflexiva realizado por quienes participan (profesorado, alumnado, o dirección por ejemplo) en las situaciones sociales (incluyendo las educativas) para mejorar la racionalidad y la justicia de: a) sus propias prácticas 
sociales o educativas; b) su comprensión sobre las mismos; y c) las situaciones e instituciones en que estas prácticas se realizan (aulas o escuelas, por ejemplo) (p.4)

Lo anterior debido a que este proyecto busca la interpretación de un aspecto social a través del uso de la investigación activa, constante y colaborativa de investigadores con el objetivo de fortalecer la comprensión lectora en los estudiantes del grado tercero primaria de la Institución Educativa Santos Apóstoles Sede Escuela Urbana de Niñas N¹9 a través de estrategias pedagógicas tales como el teatro callejero utilizándolo como medio para representar las narraciones combinando la práctica con la teoría, y usando la observación, participación, planificación y la reflexión en las narraciones y representaciones teatrales.

\section{Población}

Este proyecto se desarrolla con 73 estudiantes del grado tercero de primaria correspondientes a la jornada de la mañana del Colegio Santos Apóstoles Sede Chapinero $\mathrm{N}^{\mathrm{o}} 19$, donde 41 pertenecen al género masculino y 32 al género femenino.

\section{Instrumentos para la recolección de la información.}

Con el fin de recolectar la información necesaria para cumplir a cabalidad la investigación, se aplicaron los siguientes instrumentos: Instrumento $\mathrm{N}^{\mathrm{o}}$ 1. Diario Pedagógico. En este se registraron todas las actividades realizadas y las observaciones pertinentes a la hora de ejecutar dichas actividades. Instrumento $\mathrm{N}^{\mathrm{o}} 2$ : Rejilla diagnóstica con el fin de observar el comportamiento de los estudiantes desde la perspectiva de competencias lectoras.

\section{Resultados}

\section{Rejilla diagnóstica.}

El desarrollo de la rejilla diagnóstica se empleó por medio de la observación no participante. Para ello, se hizo un análisis de los procesos formativos en torno al área de lenguaje, se pudo estimar los hechos que componen la realidad educativa actual, dando lugar a los resultados obtenidos de la aplicación del diagnóstico, los cuales se muestran a continuación:

Un elemento de importancia se presenta a la hora de la construcción de los conocimientos, pues los estudiantes no estarían fundamentados a través de la lúdica y la creatividad. La producción de textos orales en situaciones comunicativas es mínima lo cual minimiza en gran medida el uso significativo de la entonación y articulación además de limitar su producción con un orden claro. Tales realidades pueden ser abordadas desde el uso del periódico como un instrumento cotidiano que ayuda a concretar la forma en que los estudiantes aprenden, mejorando así los hechos que componen la realidad actual de la educación.

Uno de los elementos de mayor importancia es que los estudiantes no reconocen la importancia de los hechos que componen la cultura popular, es decir no saben diferenciar entre lo que es lo cotidiano de la realidad y los hechos que dan vida a la cultura misma. Por tal motivo, no existe una vinculación académica entre ese conocimiento contextual y el académico. Donde los procesos formativos se desarrollan por medio del almacenamiento de contenido desde estructura organizativas de conocimientos un tanto tradicional. 
Tabla 1. Rejilla diagnóstica.

\begin{tabular}{|c|c|c|}
\hline $\begin{array}{c}\text { Categorías a } \\
\text { evaluar }\end{array}$ & Subcategoría & Análisis \\
\hline Metodología: & $\begin{array}{l}\text { Concepción de } \\
\text { la disciplina }\end{array}$ & $\begin{array}{l}\text { Tejedor (2001:19), hace referencia a las } \\
\text { estrategias de enseñanza y las tareas de } \\
\text { aprendizaje que el profesor propone a sus } \\
\text { alumnos en el aula, definiendo un tipo de } \\
\text { interacción didáctica. Asi pues en esta } \\
\text { intervención los conocimientos previos son } \\
\text { necesarios en cuanto a fabulas, narraciones y } \\
\text { dramatización. } \\
\text { Tejedor (2001) "no existe un método de } \\
\text { enseñanza ideal que se ajuste a todo tipo de } \\
\text { alumnos y objetivos educativos, la eficacia } \\
\text { docente dependerá de la capacidad del } \\
\text { profesor para ajustar su enseñanza a las } \\
\text { circunstancias y contexto en que se } \\
\text { desarrolla". De acuerdo a lo anterior, los } \\
\text { resultados obtenidos al realizar esta } \\
\text { intervención fueron positivos; puesto que la } \\
\text { formación está totalmente relacionada con la } \\
\text { construcción del conocimiento a través textos } \\
\text { narrativos y su posterior dramatización. }\end{array}$ \\
\hline \multirow[t]{4}{*}{ Didáctica: } & $\begin{array}{l}\text { Condiciones de } \\
\text { la enseñanza. }\end{array}$ & $\begin{array}{l}\text { Esta actividad se realizó a los estudiantes del } \\
\text { grado séptimo quienes en un } 100 \% \text { son de } \\
\text { estrato uno. }\end{array}$ \\
\hline & $\begin{array}{l}\text { Método de la } \\
\text { enseñanza. }\end{array}$ & $\begin{array}{l}\text { Roberto Abreu afirma que "el método de } \\
\text { enseñanza es un elemento movilizador de los } \\
\text { componentes cognitivos, psicomotriz y } \\
\text { afectivos de la personalidad del profesional } \\
\text { en formación en una situación concreta de } \\
\text { aprendizaje para estimular el desarrollo } \\
\text { individual y grupal" esto se ve reflejado en } \\
\text { que la actividad realizada posee los procesos } \\
\text { básicos para llegar al objetivo planteado. }\end{array}$ \\
\hline & $\begin{array}{l}\text { Importancia del } \\
\text { contexto y los } \\
\text { pre-saberes. }\end{array}$ & $\begin{array}{l}\text { Guillermina Labarrere, afirma que el método } \\
\text { de enseñanza "es la secuencia de actividades } \\
\text { del profesor y el alumno dirigidas a lograr los } \\
\text { objetivos de la enseñanza" Así pues: }\end{array}$ \\
\hline & & $\begin{array}{l}\text { Se presentó a los estudiantes la lectura: los } \\
\text { cuatro amigos, se observó apatía por leer, } 23 \\
\text { estudiantes leyeron en forma silenciosa, } 6 \\
\text { niños no leyeron el texto y } 8 \text { niños se distraían } \\
\text { mientras lo hacian, luego se procedió a leerla } \\
\text { en voz alta. }\end{array}$ \\
\hline
\end{tabular}


Se organizaron los estudiantes en grupos de lectura con el fin de realizar un guion del texto leído, se dieron las orientaciones acerca de cómo están escritos los textos dramáticos para construir en grupo el texto a representar, algunos estudiantes se confundieron al construir el guion debido a que era la primera vez que lo hacian, la docente propuso construir un guion con la participación de todos en el tablero y todos estuvieron de acuerdo, se aprovechó para explicar el género dramático y la estructura de un guion teatral y se tomó como referencia el texto leído, se inició sacando los personajes y el diálogo que le correspondia a cada uno, se buscó el vestuario (con anterioridad se les dijo que trajeran trajes de animales y otros se consiguieron en el colegio) que se fue armando para darle vida al personaje. Algunos padres de familia colaboraron con sus hijos en cuanto al vestuario.

Los estudiantes con la orientación de la docente organizaron el guion y cada grupo distribuyó los personajes. Se observó gran entusiasmo por parte de los participantes, cada cual leía una y otra vez el texto del personaje que le correspondió, se pusieron el vestuario y practicaron cada escena para la presentación de la obra, se colaboraron entre pares para el maquillaje y la creación del personaje.

Se procedió a pasar cada grupo para que dramatizaran el texto leído, se observó en los estudiantes entusiasmo en la realización de la actividad y apropiación del personaje inclusive algunos crearon voces para darle vida a cada uno. Trabajaron en equipo sobresalió el liderazgo, colaboración, solidaridad, entre otros valores.

Al terminar la presentación cada grupo planteó un fínal para la historia y la dieron a conocer a sus compañeros. Algunos propusieron que los cazadores abandonaron el bosque y los animales vivieron felices. Otro grupo propuso que el guardabosque del lugar atrapó a los cazadores y los entregó a la 
policía, otro grupo estuvo de acuerdo con el primer final.

Aprendizaje significativo (Estándares).

Pragmática

\section{Participación} del estudiante.

\section{La referencia y} la inferencia.

Niveles de
comunicación
Procesos
cognitivos.

A través de los diferentes estándares el estudiante logra desarrollar sus diferentes competencias llegando a producir textos orales en situaciones comunicativas que permiten evidenciar el uso significativo de la entonación $\mathrm{y}$ articulación. También elaborando textos escritos que respondan a diversas necesidades comunicativas y que sigan un procedimiento estratégico para su elaboración, y claramente distinguiendo las partes de un periódico y reconociendo la importancia de este medio de comunicación. Los estudiantes respondieron con agrado a la actividad ya que era un tema bastante atractivo y despertó su interés hacia la realización de actividades innovadoras.

Se hace referencia a la actividad "E1 periódico" donde cada estudiante comprende la manera como se realiza y busca la manera de dramatizarlo por medio de un magazín.

de Durante el desarrollo de la actividad se mantuvo un nivel de comunicación masiva. Los estudiantes parten de los pre saberes referentes al periódico teniendo en cuenta las siguientes preguntas:

¿Qué clase de texto es? Algunos estudiantes dijeron que era una fábula, otros 1o relacionaron a un cuento $y$ otros a una leyenda. Argumentaron el por qué observándose confusión en las clases de textos. Sin embargo 6 estudiantes lo hicieron en forma clara y sustentaron en forma correcta su respuesta.

¿Cuál es la estructura de los textos narrativos en este caso el cuento? E1 $30 \%$ de los 


\begin{tabular}{|c|c|c|}
\hline & & $\begin{array}{l}\text { estudiantes conoce la estructura de los textos } \\
\text { narrativos y las características de cada uno. }\end{array}$ \\
\hline & & $\begin{array}{l}\text { ¿Cuáles son los personajes del cuento? La } \\
\text { mayoría de los estudiantes respondieron en } \\
\text { forma correcta la pregunta pues se les leyó en } \\
\text { voz alta el texto. Sin embargo, } 6 \text { estudiantes } \\
\text { que estuvieron distraídos mientras se leía no } \\
\text { respondieron correctamente. } \\
\text { ¿Qué conclusión podemos sacar del texto? } \\
\text { Algunos estudiantes afirmaron que no tenía } \\
\text { final y otros propusieron finales como que los } \\
\text { animales del bosque vivieron muy felices, } \\
\text { otros dijeron que el guardabosque arrestó a } \\
\text { los malvados, que el león se convirtió en la } \\
\text { mascota del guardabosque. }\end{array}$ \\
\hline Impacto: & $\begin{array}{l}\text { Presentación en } \\
\text { físico de la } \\
\text { actividad. }\end{array}$ & $\begin{array}{l}\text { La actividad se presenta de manera física } \\
\text { como ficha para que los estudiantes tengan } \\
\text { claros los pasos a seguir y puedan resolver } \\
\text { cada uno de los puntos propuestos en esta. }\end{array}$ \\
\hline \multirow[t]{2}{*}{ Evaluación: } & $\begin{array}{l}\text { Rol de la } \\
\text { evaluación }\end{array}$ & $\begin{array}{l}\text { El rol de la evaluación tiene suma } \\
\text { importancia, pues se hace necesario conocer } \\
\text { la facilidad o dificultad con la que los } \\
\text { estudiantes aprenden diferentes temas, en } \\
\text { este caso, sobre medios de comunicación y } \\
\text { dramatización. }\end{array}$ \\
\hline & $\begin{array}{l}\text { Criterios } \\
\text { evaluativos }\end{array}$ & $\begin{array}{l}\text { Se evaluó la actividad, se hicieron algunas } \\
\text { observaciones acerca del tono de la voz. } \\
\text { Algunos dijeron que habían sentido mucho } \\
\text { miedo al inicio pero que después se } \\
\text { controlaron. De este modo, la expresión de } \\
\text { las emociones, realizadas por medio de la } \\
\text { redacción y argumentación, propicio un } \\
\text { excelente el trabajo responsable de los niños } \\
\text { fue muy exitoso, y sobre todo les gustó } \\
\text { mucho actuar, según ellos se habían } \\
\text { transportado al bosque y habían sentido lo } \\
\text { que el personaje vivía, que había sido muy } \\
\text { bonito actuar }\end{array}$ \\
\hline
\end{tabular}

\section{Rejilla de Evaluación.}

El desarrollo de la rejilla de evaluación se llevó a cabo mediante la aplicación de la observación, de este modo se pudo establecer los hechos que componen la realidad, en torno a los procesos de enseñanza de la competencia comunicativa y de los textos narrativos a través del teatro callejero. Por otra parte, la realidad 
educativa actual apunta a que es necesario reestructurar la educación para que deje el sentido tradicional adquirido y comience a sentar las bases a través de la didáctica que en este caso fueron:

El resultado principal se centra en reconocer que los estudiantes comprenden mejor a través del desarrollo de procesos formativos fundamentados en el teatro callejero; de este modo, se puede desarrollar la capacidad lúdica y critica de estos con la incorporación de la competencia comunicativa. Es por ello, que incluso los procesos de comunicación oral, así como la capacidad de los estudiantes para emprender el desarrollo de discursos orales mejoro, al incluir aspectos como entonación y articulación para una mejor expresión textual. Por otra parte, el uso del periódico como un documento informativo y compuesto en su estructura de elementos literarios permitió que se destacara su importancia a la hora de comunicar, se percibe un cambio significativo a la hora en que los estudiantes emprenden sus labores académicas.

Tabla 2. Rejilla de evaluación.

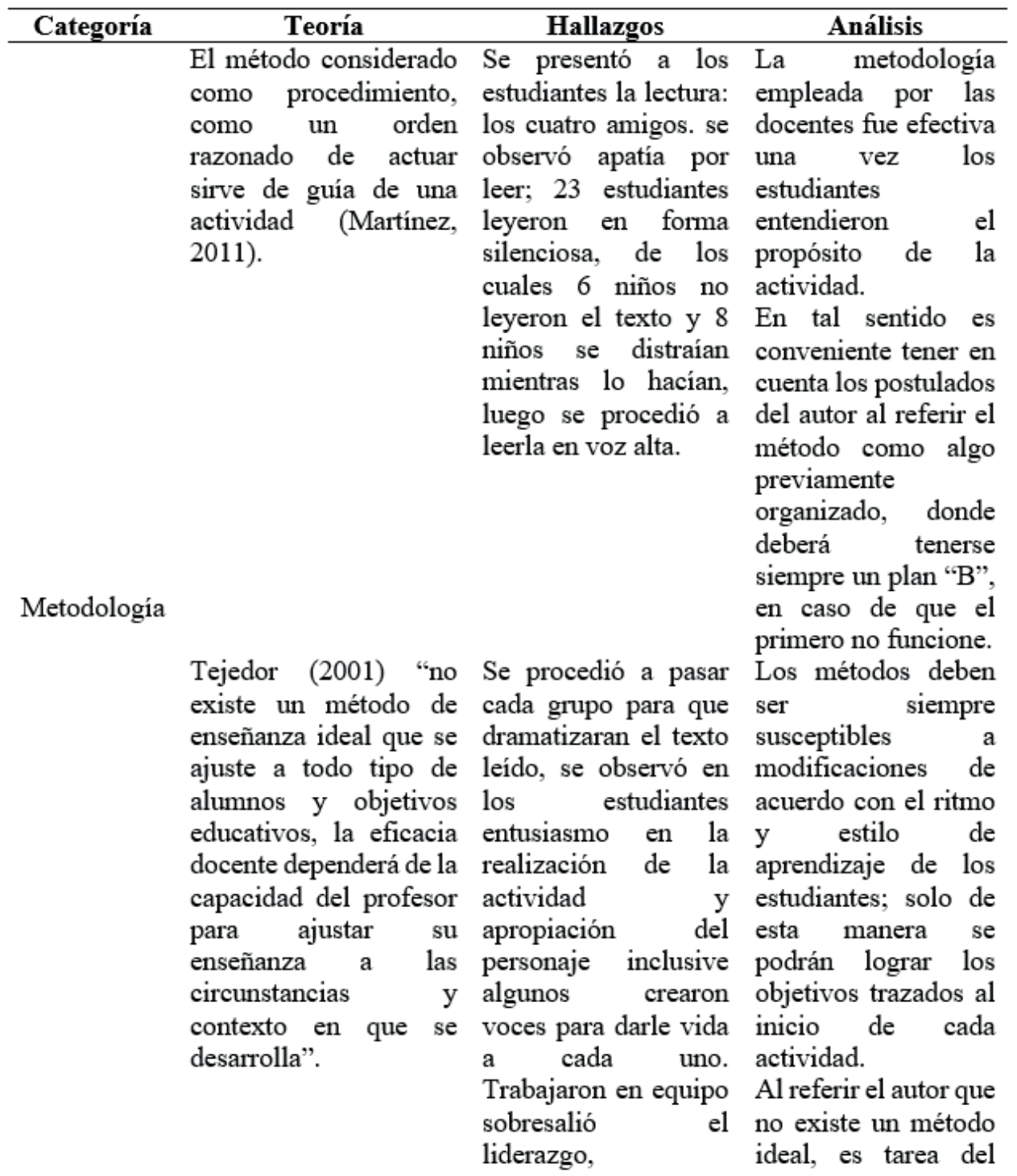


Didáctica
Roberto Abreu afirma que "el método de enseñanza es un elemento movilizador de los componentes cognitivos, psicomotriz $y$ afectivos.

Roberto Abreu afirma
que "el método de
enseñanza es un
elemento movilizador
de los componentes
cognitivos, psicomotriz
y afectivos.

colaboración, solidaridad, otros valores. diferentes estándares los estudiantes lograron desarrollar competencias

llegando a producir textos orales en diferentes situaciones comunicativas
A través de los

docente encontrar aquel que se ajuste a las necesidades y expectativas del grupo de turno.

E1 docente siempre debe tener presente, en el diseño de su método, la forma en que el estudiante adquirirá el aprendizaje y hacer énfasis en ello una vez este aplicándolo. Por otra parte, ha de tener en cuenta la formación integral, incluyendo en sus actividades aspectos cognitivos, psicomotrices $y$ afectivos.

Guillermina Labarrere, Se organizaron los afirma que el método de estudiantes en grupos enseñanza "es la de lectura con el fin secuencia de de realizar un guion actividades del profesor del texto leído, se y el alumno dirigidas a lograr los objetivos de la enseñanza".

dieron las orientaciones acerca de cómo están escritos los textos dramáticos para construir en grupo el texto a representar.

Según (Sarramona, Los estudiantes 1998), una competencia estuvieron muy se vincula con las entusiasmados por capacidades más que consultar sobre el con simple saberes, porque el énfasis se pone en el <saber hacers, sin que falten por ello los saberes y las predisposiciones $o$ actitudes que los envuelven.
De acuerdo con los planteamientos de la autora, se infiere que la estrategia elegida para el desarrollo de un tema debe ser claro y conciso, sugiriendo a los estudiantes seguir la secuencia planteada para de esta manera lograr los objetivos propuestos.

Del cruce de la información se infiere que el docente debe dar luz verde a la creatividad de sus estudiantes $y$ motivarlos a tener una buena actitud ante cualquier actividad académica. Por otra parte, es importante valorar cualquier trabajo o actividad que realice el estudiante, pues 
Pragmática

(Colombia Aprende,
2012), expresa que la
competencia
comunicativa - lectora
explora la forma como
los estudiantes leen e
interpretan diferentes
tipos de textos. Se
espera que puedan
comprender tanto la
información explícita
como la implicita en los
textos, establecer
relaciones entre sus
contenidos y lo que
saben acerca de un
determinado tema, así
como realizar
inferencias, sacar
conclusiones y asumir
posiciones

E1 (MEN, 2015) menciona que el partieron de los pre maestro debe diseñar $\mathrm{e}$ implementar estrategias pedagógicas que le permitan potenciar el trabajo que hace en el aula $y$ que pueda brindar herramientas de calidad para enseñanza - aprendizaje

aje

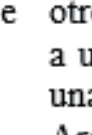
Argumentaron el por Argumentaron el por qué, observándose confusión en las clases de textos. Sin embargo estudiantes 6 hicieron en forma clara y sustentaron en forma correcta su respuesta.

Los estudiantes con la orientación de la docente organizaron el guion y cada grupo distribuyó los personajes. $\quad \mathrm{Se}$ observó gran entusiasmo por parte de los participantes, cada cual leía una y otra vez el texto del personaje que le correspondió. $\mathrm{Se}$ pusieron el vestuario $y$ practicaron cada escena para la presentación de la obra. Se colaboraron entre pares para el maquillaje y
creación personaje. ello lo llenará de motivos para seguir trabajando y perfeccionarse cada vez más.

Para este caso, los docentes parten de los presaberes de los estudiantes para formar un nuevo concepto, lo cual permite que el aprendizaje sea más fluido y la actividad al interior del aula más amena.

Cuando el docente tiene en cuenta los conocimientos previos del estudiante, su clase se convierte en un diálogo ameno y entretenido para los dos partes.

Cuando los
estudiantes han
desarrollado su
competencia lectora,
son capaces de
apropiarse de un
texto y llevarlo a un
contexto personal,
social o academico.
En este caso los
estudiantes se
apropiaron del guion
que le correspondió a
cada uno, logrando
dar vida a sus
personajes de
acuerdo con el
análisis y la
interpretación que le
dieron a cada uno.
Todo lo mencionado
hace un aporte


argumentadas frente a los mismos. significativo al trabajo colaborativo, pues en una actividad de este tipo es necesario una sinergia completa entre todos los protagonistas.

(Fonseca, 2007) Las Se observó interés y Al referirse el autor estrategias didácticas entusiasmo por al trabajo apuntan a fomentar participar en la colaborativo, se procesos de dramatización, todos detecta una relación autoaprendizaje, los integrantes se aprendizaje interactivo y aprendizaje colaborativo.

Martínez, 2011). Se facilitó la Mientras se pone en actividad pues se práctica la estrategia, trataba de algo que les todas las acciones gustaba, de sus tienen un sentido, una intereses, aunque orientación. colaboraron

buscando vestuario, cámaras y micrófonos para la presentación, los grupos se unieron para la escenografia y vestuario prestándose los materiales entre si. fortalecer el tono de la voz, mejoró un poco en comparación a la actividad anterior. Los niños respondieron con con Lo anterior se agrado a la actividad traduce en que la 


\begin{tabular}{ll} 
ya que era un tema de & estrategia debe \\
su interés. & contemplar \\
& momentos que \\
permitan constatar \\
& que el trabajo se esté \\
& realizando y que se \\
& esté haciendo de \\
& manera correcta; de \\
& esta manera se podrá \\
& garantizar la \\
& efectividad de la \\
& actividad planteada. \\
\hline
\end{tabular}

\section{Conclusiones y recomendaciones}

Los elementos relevantes en torno a la aplicación de la investigación, giran en torno a tratar de entender la forma como los docentes específicamente los dedicados a enseñar en el área de lenguaje desarrollan sus clases. La realidad inminente señala que el tradicionalismo es un factor de gran influencia en el acto educativo, es decir, se sigue enseñando desde dimensiones netamente teóricas, no es que no sea necesario el dominio de información específica del área, más bien, es necesario desde lo planteado, que se trate de involucrar una serie de elementos prácticos que sirvan de elementos dinamizadores del proceso de enseñanza. La inclusión de nuevas tendencias del pensamiento didáctico en el que hacer educativo; representaría una alternativa de cambio que involucre a la educación en nuevos retos didácticos tal cual y como se afirmó a lo largo de la presente investigación.

Por ello, se evidencia y concluye que, en el ámbito educativo, específicamente en el contexto abordado, las perspectivas de enseñanza del área de lenguaje, siguen enmarcadas en posturas tradicionales y poco innovadoras, factor que influye de forma directa en el proceso de enseñanza incidiendo en el desarrollo académico de los estudiantes en torno al desarrollo de la comunicación.

En relación al manejo de la apropiación de la competencia comunicativa para la enseñanza se pudo evidenciar que es necesario el uso de los textos narrativos por medio del teatro callejero como estrategia sustentada en los modelos pedagógicos actuales para el desarrollo de los hechos propuestos por el MEN para el área de lenguaje.

La situación antes planteada genera gran preocupación, pues para la comprensión y consolidación de los conocimientos a través de la competencia comunicativa, donde lo que se pretende es que los docentes manejen asertivamente la competencia comunicativa a través de los textos narrativos por medio del teatro callejero para poder reestructurar las realidades educativas actuales. Al presentarse debilidades en la competencia comunicativa, se generan vacíos conceptuales y procedimentales que afectan la adquisición y construcción de conocimientos por parte del estudiante, es allí donde cobra un valor importante el uso del teatro callejero. Los resultados antes expuestos, permiten inferir que las estrategias empleadas por los docentes en torno al desarrollo de la competencia comunicativa están centradas en prácticas pedagógicas que promueven el aprendizaje memorístico y repetitivo, sin generar procesos de reflexión y comprensión numérica a partir de los conocimientos impartidos en el aula. 
Es evidente, que desde esta perspectiva se deben replantear los procesos de enseñanza y de aprendizaje en dicha área del conocimiento a fin de promover el desarrollo de procesos cognitivos de mayor complejidad en el estudiante para fortalecer las competencias necesarias y básicas para la construcción de conocimientos para la vida. La realidad educativa incluya nuevas tendencias que signifique el avance del hecho educativo, con la inclusión del teatro callejero como una forma de enseñanza activa que le permitirán al docente realizar procesos de análisis, reflexión y transferencias de conocimientos a partir de las necesidades educativas de los estudiantes.

En virtud de lo antes expuesto, surgen retos hacia la orientación de una educación distinta que debe fundamentarse en la contextualización y experimentación para que el estudiante aprenda haciendo y viviendo desde su propia realidad, permitiendo que el docente vaya incluyendo e incorporando elementos metodológicos propios de la cotidianidad, para que logre consolidar un aprendizaje significativo. Por tal motivo, el uso de los textos narrativos por medio del teatro callejero se presenta como una herramienta oportuna para el manejo de la competencia comunicativa.

\section{Referencias}

Acosta, A. (2007). Cómo analizar los tipos de discurso. Madrid: Autor-Editor.

Adam, J. M., \& Lorda, C. (1999). Lingüística de los textos narrativos. Barcelona: Ariel.

Álamo, F. (2016). Las relaciones extratextuales e intratextuales entre texto narrativo y autor. Teoría y modelos.

Avanzini, G. (1998). La pedagogía hoy. México D.F.: FCE.

Betancourth, M., \& Madrodeño, E. (2014). La enseñanza para la comprensión como didáctica alternativa para mejorar la interpretación y producción oral y escrita en lengua castellana en el grado quinto del centro educativo municipal la victoria de Pasto. Pasto: Universidad de
Manizales.

Colombia Aprende. (2010). Programa para el desarrollo de competencias. Bogotá: Ministerio de Educación Nacional.

Colombia Aprende. (2012). Plan Nacional de Lectura y Escritura. Bogotá: Ministerio de Educación.

Constitución Política de Colombia. (2001). Art 67. Bogotá: Temis. Recuperado el 23 de 1 de 2017

Daniels, H. (2001). Gender and learning: equity, equality and pedagogy. SFL, 16(3), 112-116. doi:10.1111/1467-9604.00201

Duque, C., \& Correa, M. (2012). Inferencias sobre un texto narrativo en contextos de interacción en la educación inicial. Revista Javeriana, 11 (ISSN 1657-9267), 559-570.

EACEA. (2011). La enseñanza de la lectura en Europa: contextos, políticas y prácticas. Bruselas: Agencia Ejecutiva en el ámbito Educativo, Audiovisual y Cultural. Recuperado el 18 de 01 de 2017, de http://eacea.ec.europa. eu/education/eurydice

Escate, G. (2012). Niveles de comprensión lectora en alumnos de cuarto grado de primaria de Carmen de la legua Reynoso - callao. LimaPerú: Universidad San Ignacio de Loyola.

Fonseca, M. (2007). "Enseñar en la universidad. Experiencias y propuestas de docencia universitaria. La Coruña: Netbiblo.

Fraca, L. (2008). Una pedagogía integradora estratégica a partir de un manual de ortografía. Barcelona: El Nacional.

González G. (2007). El teatro callejero: fenómeno de comunicación que puede hacer uso de las nuevas tecnologías para formar en valores. Posgrado y Sociedad Vol. 7 No. 1.

Hernández, G. (2008). Paradigmas en psicología de la educación. Ecuador: Paidós. Recuperado el 22 de 1 de 2017

Herrera, B. (2010). Aplicación de estrategias de lectura para la potenciación de la comprensión lectora utilizando el texto electrónico como recurso didáctico en el primer nivel superior de unitec. Tegucigalpa: universidad pedagógica 
nacional francisco Morazán.

Hurtado, M., \& Ortiz, R. (2012). Fortalecimiento de la comprensión lectora a partir del texto narrativo fábula en los estudiantes del grado cuarto de los Centros Educativos Salamina-Sede Naranjales, del municipio de Currillo y Teusaquillo- Sede Nueva Zelanda. Florencia, Caquetá: municipio de Cartagena del Chaira.

León, J. A. (2014). La mejora de la comprensión lectora: un análisis interactivo. Journal for the Study of Education and Development, 14(Issue 56), 3-24. Recuperado el 18 de 12 de 2016, de http://dx.doi.org/10.1080/02103702.1991.1082 2312

Ley 115. (1994). Educación informal. Bogotá: Congreso De La República De Colombia.

Llanos, O. (2013). Nivel de compresión lectora en estudiantes de primer ciclo de carrera universitaria. lima: universidad de Piura. recuperado el 15 de 12 de 2016, de https://pirhua. udep.edu.pe/bitstream/handle/123456789/1814/ MAE_EDUC_106.pdf?sequence $=1$

Madera, I. P., \& Gómez, L. F. (Marzo de 2013). El proceso de comprensión lectora en alumnos de tercero de secundaria. Revista Mexicana de Investigación Educativa, 18(56), 113139. Obtenido de http://www.redalyc.org/ pdf/140/14025581006

Martínez, E. (2011). Las estrategias y técnicas didácticas en el rediseño. Monterrey: Dirección de Investigación y Desarrollo Educativo.

MEN. (2003). Bogotá: Ministerio de Educación Nacional. Recuperado el 23 de 1 de 2017, de http://www.mineducacion.gov.co/1621/ articles-86408_Archivo_pdf.pdf

MEN. (2006). Estándares Básicos de Competencias en Lenguaje, Matemáticas, Ciencias y Ciudadanas. Colombia: Ministerio de Educación Nacional. Recuperado el 12 de 01 de 2016, de http://www.mineducacion.gov.co/1621/ articles-340021_recurso_1.pdf

MEN. (2015). De los responsables y sus competencias generales en el marco del proceso de gestión de la cobertura educativa. Bogotá:
Ministerio de Educación Nacional.

Prieto, D. (2016). Propuesta metodológica para potenciar las competencias escriturales, por medio de la narrativa digital en estudiantes de octavo grado del colegio Alexander Fleming $G$ I.E.D. Bogotá: Universidad Libre.

Sarramona, J. (1998). Teoría de la educación. Madrid: Ariel.

Velasco, M., \& Tabares, L. (2015). La comprensión de textos narrativos: implementación de una secuencia didáctica de enfoque comunicativo, con estudiantes de grado segundo. Pereira: Universidad Tecnológica de Pereira. 